\title{
Current use of silkworm larvae (Bombyx mori) as an animal model in pharmaco-medical research
}

\author{
Don Daniel Nwibo ${ }^{1}$, Hiroshi Hamamoto ${ }^{1}$, Yasuhiko Matsumoto ${ }^{1}$, Chikara Kaito ${ }^{1}$, \\ Kazuhisa Sekimizu ${ }^{1,2, *}$ \\ ${ }^{1}$ Laboratory of Microbiology, Graduate School of Pharmaceutical Sciences, The University of Tokyo, Tokyo, Japan; \\ ${ }^{2}$ Genome Pharmaceuticals Institute, Tokyo, Japan.
}

\begin{abstract}
Summary We advocate the use of silkworm larvae, Bombyx mori, as an animal model for discovery of drug candidates. We have established several disease models using silkworms, which offer technical advantages in drug development and the study of host-pathogen interaction. This mini review briefly describes recent trends in the use of silkworm larvae as a nonmammalian model for drug discovery and it offers suggestions regarding the potential for silkworm use in pharmaceutical-biomedical research.
\end{abstract}

Keywords: Animal model, silkworm, drug discovery

\section{Merits of silkworm models}

More often than not, evaluation of drug candidates involves problems deciding which animals to use. These problems can be classified into ethical issues in terms of animal welfare, cost, and ease of maintenance $(1,2)$. In Asia and Africa, little attention has been paid to ethical issues. However, research bodies and institutions around the world have committees that oversee experimental use of animals. The guiding rationale behind rules regulating animal use are the potential benefit to human health, minimized pain or distress to animals, and use of timely and humane procedures for euthanasia based on the principles of Replacement, Reduction, and Refinement (3-5). Problems with animal use during evaluation of the therapeutic effects of pharmaceuticals can be avoided through use of alternative animal models, and especially non-mammalian models. Non-mammals, such as Caenorhabditis elegans and Drosophila melanogaster, have been used as models (6-9). However, these animals are too small to handle and are thus unsuitable for injecting an accurate volume of a test sample, which is a technical requirement for in vivo experiments. Therefore, the use of silkworm larvae, Bombyx mori, may represent a way to

\footnotetext{
*Address correspondence to:

Dr. Kazuhisa Sekimizu, Laboratory of Microbiology, Graduate School of Pharmaceutical Sciences, The university of Tokyo, 7-3-1 Hongo, Bunkyo-ku, Tokyo 113-003 Japan.

E-mail: sekimizu@mol.f.u-tokyo.ac.jp
}

overcome these problems. Courtesy of sufficient size, silkworm larvae offer a number of advantages, such as facilitation and accuracy of intra-hemolymph injection (corresponding to intravenous injection in mammals), the possibility of intra-midgut injection (corresponding to oral administration in mammals), and the ease of organ isolation for research (10-13). Moreover, typical physical changes (like variation in color, a reduction in size or elongation, and slowed movement) are readily apparent when comparing these phenotypes with those of the controls. One need not wait for larvae to die before making reproducible scientific inferences, especially in time or dose-dependent experiments.

\section{Evaluation of the therapeutic effect of antibiotics}

Silkworm larvae have been used to evaluate the virulence of human pathogenic bacteria. For instance, previous reports have shown that $S$. aureus, $P$. aeruginosa, and $V$. cholera, were lethal to silkworm models $(12,13)$. The current authors' research group has used a silkworm model of infection to evaluate S. aureus virulence factors $(14,15)$. The agr locus, a hemolysin gene regulator, was found to contribute to the pathogenicity of $\mathrm{S}$. aureus in animal hosts (15). Silkworm models have also been used to study other virulence factors, including bacterial toxins and hemolysins (16). By extension, this means that silkworm is an effective model organism for the study of host-pathogen interaction and genetic changes and virulence factors of pathogens. 


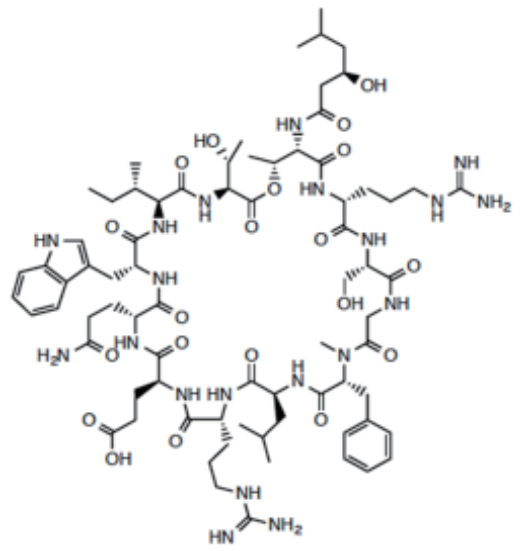

Figure 1. Structure of lysocin $\mathbf{E}$ discovered using a silkworm model of infection (20). A cyclic-lipopeptide, lysocin $\mathrm{E}$ is a novel antibiotic that is effective against Gram-positive bacteria including methicillin-resistant Staphylococcus aureus (MRSA).

Hamamoto et al. using silkworm models to quantitatively evaluated the therapeutic effects of known antibiotics (including vancomycin, kanamycin, chloramphenicol, flomoxef, teicoplanin, tetracycline, minocycline, arbekacin, and linezolid), and they reported that in the model drugs had an $\mathrm{ED}_{50}$ against human pathogenic bacteria (Staphylococcus aureus and Stenotrophomonas maltophilia) and true fungi (Candida albicans and Candida tropicalis) that was consistent with the $\mathrm{ED}_{50}$ when those drugs were administered to mice (11). In a silkworm model of infection, orally administered antibiotics displayed potency similar to that displayed in larger animals. A study also noted that the silkworm model was suitable for evaluation of the toxicity and metabolism of therapeutic drug candidates (17). Silkworm models, therefore, offer a more economic means of testing the effectiveness of antibiotics. Nosokomycin, a derivative of moenomycin A from Streptomyces sp. K04-0144, was discovered in 2010 in a silkworm model of MRSA infection (18). More recently, lysocin E (Figure 1), a bactericidal antibiotic from the cultured supernatant of the soil bacterium Lysobacter sp. RH2180-5, was discovered in a silkworm model of infection and was found to target menaquinone, a component of the electron-transport chain in the membrane of Gram-positive bacteria (19). Furthermore, a silkworm model of infection is an excellent model with which to exclude non-therapeutic molecules.

\section{Models to evaluate anti-diabetic drugs}

During the development of hyperglycaemic silkworm model for anti-diabetic drug screening, Matsumoto et al. $(20,21)$ reported an immediate rise in the hemolymph sugar level (corresponding to blood glucose in mammals) in larvae fed a high glucose diet. Larvae fed a high glucose diet were found to suffer from hyperglycemia-related disorders, including reduced

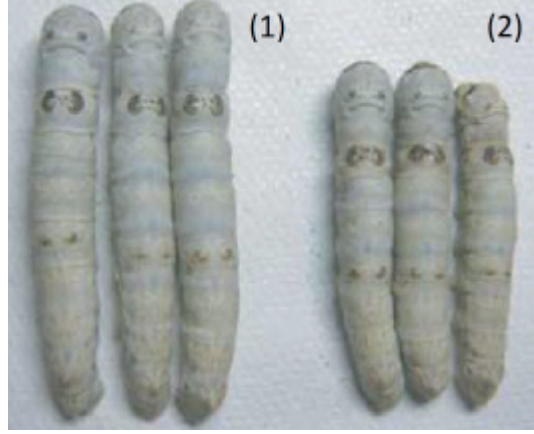

Figure 2. Hyperglycemic silkworms show growth defects (21). $5^{\text {th }}$ instar larvae fed a normal diet and injected with (1) saline or (2) glucose solution.

body size (Figure 2). Much like mammals, silkworms have been shown to possess a regulatory system for hemolymph sugar homeostasis (22). The antihyperglycemic effect of known anti-diabetic drugs was successfully evaluated using hyperglycemic silkworm model (20). The administration of recombinant human insulin led to a decrease in hemolymph sugar levels in larvae fed a high glucose diet and consequently restored growth (20). The AMPK-signaling pathway plays an important role in maintaining systemic glucose levels in larger animals, and this pathway was also found to function in hyperglycemic silkworms (20).

'Humanized silkworms', which refer to transgenic silkworms expressing human insulin receptors, have also been used to evaluate therapeutically active insulin receptor agonists (21). Administration of human insulin to humanized silkworms decreased hemolymph sugar levels and facilitated phosphorylation of $A k t$, which is a key factor of insulin-signaling pathway, in the fat body. Therefore, silkworms are not only useful animals but also are also simpler, cheaper, and easier to handle when evaluating anti-diabetic drug candidates. Moreover, silkworm models could be used to study other human metabolic disorders, such as obesity and hypertension, given the similarities in the pathophysiological mechanisms of silkworm and larger animals.

\section{Prospects for the future and potential challenges}

Silkworm larvae represent a satisfactory model for discovery and evaluation of therapeutic substances. The aforementioned findings demonstrate that silkworms compare favorably to other animal models. However, certain areas of pharmaco-medical research, such as those involving neurological disorders and aging, have yet to embrace the use of silkworm larvae as animal models. One argument against the use of silkworms in such studies is because larvae do not contract the same genetic diseases as humans. Therefore, genetic engineering techniques (biotechnology procedures to manipulate the silkworm genome using gene knock- 
outs and knock-ins) have been established (10), and silkworm genes could be manipulated to induce such disease states. Thus, diseases not naturally contracted by insects could be successfully studied using genetically engineered silkworms. In this way, silkworm via genetic modification present scientists with models for addressing mechanistic questions and molecular targets in drug discovery research.

\section{References}

1. Neil L. The use of animal as models: Ethical considerations. Int J Stroke. 2012; 7:440-442.

2. Monamy V. Animal Experimentation. A Guide to the Issues. Cambridge, Cambridge University Press, 2009.

3. Principles of Laboratory Animal Science (Van Zutphen LFM, Baumans V, Beynen AC, eds.), Elsevier Amsterdam, 2001; pp. 219-250.

4. Weisser K, Hechler U. Animal Welfare Aspects in the Quality Control of Immunobiologicals. A Critical Evaluation of Animal Tests in Pharmacopoeial monographs. London: Nottingham, FRAME, 1997.

5. Hendriksen CFM. Refinement, reduction, and replacement of animal use for regulatory testing: Current best scientific practices for the evaluation of safety and potency of biologicals. ILAR J. 2002; 43(Supp1 1):S43-S48.

6. Mahajan-Miklos S, Tan MW, Rahme LG, Ausubel FM. Molecular mechanisms of bacterial virulence elucidated using a Pseudomonas aeruginosa-Caenorhabditis elegans pathogenesis model. Cell.1999; 96:47-56.

7. Hamilton PJ, Campbell NG, Sharma S, Erreger K, Hansen FH, Saunders C, Belovich AN, Sahai MA, Cook EH, Gether U, Mchaourab HS, Matthies HJG, JS Sutcliffe JS, Galli A. Drosophila melanogaster: A novel animal model for the behavioral characterization of autism-associated mutations in the dopamine transporter gene. Mol Psychiatr. 2013; 18:1235.

8. Peterson R, Nass R, Boyd WA, Freedman JH, Dong K, Narahashi T. Use of non-mammalian alternative models for neurotoxicological study. Neurotoxicology. 2008; 29:546-555.

9. Shalina MM, Laurence GR, Frederick MA. Elucidating the molecular mechanisms of bacterial virulence using non-mammalian hosts. Mol Microbio. 2000; 37:981-988.

10. Tamura T, Thibert C, Royer C, Kanda T, Eappen A, Kamba M, Kômoto N, Thomas JL, Mauchamp B, Chavancy G, Shirk P, Fraser M, Prudhomme JC, Couble P. Germline transformation of the silkworm Bombyx mori L. using a piggyBac transposon-derived vector. Nat Biotechnol. 2000; 18:81-84.

11. Hamamoto H, Kurakawa K, Chikara K, Kamura K, Razanajatovo M, Iony KH, Santa T, Sekimizu K. Quantitative evaluation of the therapeutic effects of antibiotics using silkworm infected with human pathogenic microorganisms. Antimicrob Agents Chemother. 2004; 48:774-779.

12. Kaito C, Sekimizu K. A silkworm model of pathogenic bacterial infection. Drug Discov Ther. 2007; 1:89-93.

13. Kaito C, Akimitsu N, Watanabe H, Sekimizu K. Silkworm larvae as an animal model of bacterial infection pathogenic to humans. Journal of Microbial Pathogenesis. 2002; 32:183-190.

14. Kaito C, Kurokawa K, Matsumoto Y, Terao Y, Kawabata S, Hamada S, Sekimizu K. Silkworm pathogenic bacteria infection model for identification of novel virulence genes. Mol Microbiol. 2005; 56:934-944.

15. Miyazaki S, Matsumoto Y, Sekimizu K, Kaito C. Evaluation of staphylococcus aureus virulence factors using a silkworm model. FEMS Microbiol Lett. 2011; 326:116-124.

16. Hossain MS, Hamamoto H, Matsumoto Y, Razanajatovo IM, Larranaga J, Kaito C, Kasuga H, Sekimizu K. Use of silkworm larvae to study pathogenic bacterial toxins. J Biochem. 2006; 140:439-444.

17. Hamamoto H, Tonoike A, Narushima K, Horie R, Sekimizu K. Silkworm as a model animal to evaluate drug candidate toxicity and metabolism. Comp Biochem Physiol C Toxicol Pharmacol. 2009; 149:334-339.

18. Uchida R, Iwatsuki M, Kim YP, Ohte S, Omura S, Tomoda H. Nosokomycins, new antibiotics discovered in an in vivo-mimic infection model using silkworm larvae. I: Fermentation, isolation and biological properties. J Antibiot (Tokyo). 2010; 63:151-155.

19. Hamamoto H, Urai M, Ishii K, et al. Lysocin E is a new antibiotic that targets menaquinone in the bacterial membrane. Nat Chem Biol. 2015; 11:127-133.

20. Matsumoto Y, Sumiya E, Sugita T, Sekimizu K. An invertebrate hyperglycemic model for the identification of anti-diabetic drugs. PLoS ONE. 2011; 6:e18292.

21. Matsumoto Y, Ishii M, Ishii K, Miyaguchi W, Horie R, Inagaki Y, Hamamoto H, Tatematsu T, Uchino K, Tamura T, Sezutsu H, Sekimizu K. Transgenic silkworms expressing human insulin receptors for evaluation of therapeutically active insulin receptor agonists. Biochem Biophys Res Commun. 2014; 455:159-164.

22. Chitra C, Sridhara S. Transport of sugars by the fat body of silkworm, Bombyx mori. J Insect Physiol. 1973; 19:2053-2061.

(Received April 16, 2015; Accepted April 24, 2015) 